\title{
Problematización epistémica de la pedagogía: Algunas definiciones y aproximaciones
}

Maximiliano Heeren Herrera. Universidad Católica de Temuco

Recepción: 01.12.2018 | Aceptado: 15.10.2019

Correspondencia a través de ORCID: Maximiliano Heeren Herrera

iD $\quad 0000-0001-7056-7350$

Citar: Heeren Herrera, M. (2019). Problematización epistémica de la pedagogía: Algunas definiciones y aproximaciones. REIDOCREA, 8,133-141.

Resumen: Este artículo tiene como objetivo evidenciar una aproximación respecto de la problematización de una episteme de la pedagogía a través de las definiciones que se han hecho de ella y la exposición de algunas teorías educativas que forman parte del recorrido epistemológico de la pedagogía. Ello permitirá contribuir con la discusión epistémica acerca de la elaboración de conocimiento en el campo de la pedagogía. Metodológicamente, se llevó a cabo una revisión conceptual del término pedagogía en artículos de divulgación científica a nivel internacional y nacional, en relación con algunas teorías educativas que tienen a la base una forma de construir conocimiento que pudiese generar aportes o aproximaciones en la comprensión de la construcción de conocimiento desde la pedagogía. Los resultados de esta revisión revelan que a lo largo de la historia de la pedagogía se han desarrollado maneras de concebir al mundo y a raíz de ella, se ha construido conocimiento en el ámbito pedagógico. La conclusión que subyace a esta reflexión es que la pedagogía debe ser comprendida desde el campo epistémico en relación con el saber pedagógico, pues se ha construido conocimiento desde los inicios de ésta a través de sus definiciones y su que hacer en el campo educativo.

Palabras clave: Epistemología | Pedagogía

Epistemic problem of pedagogy: some definitions and approximations

Summary: The aim of this article is to demonstrate an approach to the problematization of an episteme of pedagogy through the definitions that have been made of it and the exposition of some educational theories that are part of the epistemological journey of pedagogy. This will allow contributing to the epistemic discussion about the elaboration of knowledge in the field of pedagogy. Methodologically, a conceptual review of the term pedagogy was carried out in articles of scientific divulgation at international and national level, in relation to some educational theories that have at the base a way of constructing knowledge that could generate contributions or approximations in the understanding of the construction of knowledge from pedagogy. The results of this review reveal that throughout the history of pedagogy, ways of conceiving the world have been developed and, as a result, knowledge has been built in the pedagogical sphere. The conclusion that underlies this reflection is that pedagogy must be understood from the epistemic field in relation to pedagogical knowledge, since knowledge has been built from the beginning of it through its definitions and its task in the educational field

Keywords: Epistemology | Pedagogy

\section{Introducción}

El presente artículo se propone evidenciar cómo la pedagogía desde su origen hasta la actualidad se estructura a través del saber, el discurso, la disciplina y los mensajes implícitos y cómo por medio de ellas podría construir su propio estatus epistemológico. El contenido del ensayo se estructura; en primer lugar, en visibilizar algunas definiciones pedagógicas que tienen a la base el saber, el discurso, la disciplina y los mensajes implícitos para constituirse como una epistemología. Ante ello, de igual manera, se propone conocer la forma en que se instala la construcción de un estatus epistemológico de la pedagogía por medio de algunos autores que han desarrollado dicha construcción. En síntesis, aproximarnos a cómo es que se estaría diseñando una epistemología de la pedagogía, considerando que, actualmente, la pedagogía para constituirse como ciencia tendrá que responder varias preguntas, entre ellas, qué es y lo que no es pedagogía, su objeto de estudio, entre otras interrogantes. 


\section{Problema de estudio}

El problema que plantea este ensayo se vierte en la compleja situación en la que se encontraría una construcción epistemológica de la pedagogía, debido a las múltiples definiciones que tendría que establecer para constituirse epistemológicamente.

En una primera mirada se evidencia el problema que presentan los positivistas y neopositivistas al negarle el estatus científico a la pedagogía por carecer de leyes y teorías propias, pues solo crearía procedimientos que estarían fundados en otras ciencias auxiliares (Hermoza, 2011).

Una propuesta a este problema sería la siguiente forma de abordar la pedagogía como una ciencia:

1.- Asumir una perspectiva cualitativa, en la cual se piense a la pedagogía desde la comprensión, la crítica y la transformación de los sujetos, es decir, considerar un paradigma hermenéutico y sociocrítico.

2.- Considerar algunas características que cumple la pedagogía para constituirse como ciencia (Loayza, 2009).

Lo anterior remite, necesariamente, a las definiciones que hoy se hacen respecto de la pedagogía como saber, discurso, disciplina y mensajes implícitos, las cuales son necesarias para comprender cómo es que se está llevando a cabo la construcción de una episteme de la pedagogía. La explicitación de cada una de estas definiciones será abordada en este artículo, de igual forma, se presentará una propuesta de construcción de una epistemología de la pedagogía en la conclusión de este estudio, a partir de las definiciones dadas.

\section{Definiciones de pedagogía}

A continuación, se presentarán cada una de las distintas definiciones asignadas a la pedagogía. Lo anterior abordará la pedagogía como saber, discurso, disciplina y mensajes implícitos a través de distintos autores, además se irá complementando con algunas teorías educativas que consideran el concepto en el proceso de enseñanza aprendizaje.

\section{Pedagogía como un saber}

En la construcción de una epistemología de la pedagogía, muchos autores han intentado constituir su estatus a través del saber pedagógico, tanto es así, que algunos teóricos señalan que la epistemología de la pedagogía estaría dada por el saber pedagógico que en muchos casos se encontraría de forma implícita al resolver las coyunturas que se pueden suceder en el aula (Patiño y Rojas, 2009). En relación a ello se ha señalado que toda disciplina científica se organiza en torno a una episteme que estructura los saberes y discursos de una época (Foucault, 1982, 1997), por lo tanto, si la pedagogía anhelase constituirse como ciencia debería estar estructurada a través de los saberes. Ello, porque un saber está construido bajo un contexto social, en el cual se asume de forma inherente una historicidad y cultura (Beillerot, 1998). Lo anterior será de vital importancia para comprender lo que ocurre en el escenario educativo. En dicho espacio será el docente quien asuma su quehacer pedagógico en tanto conocimientos y saberes que provienen de él y será a través de ellos que ira estructurando y orientando su práctica (Tardif, 2004). En este sentido, si el docente es quien va estructurando su saber, será en las relaciones que pueda establecer dentro y fuera del aula donde encuentre dichos 
saberes, que en lo experiencial lo conducen a tener una posición ante la construcción del conocimiento. Al respecto, se instala con fuerza la concepción de que es en la acción pedagógica, que está en relación con distintos escenarios socioculturales, donde se construye el saber pedagógico (Muñoz, Quinteros y Munévar, 2002).

En relación con lo anterior, el saber pedagógico sería inherente en la acción (Latorre, 2004), es decir, se construiría en el quehacer pedagógico y se constituiría en una metareflexión del docente (Mena y Romagnoli, 1993). Al establecer estas relaciones cobra sentido la existencia de una episteme de la pedagogía en directa relación con la existencia de un saber pedagógico (Noguera, 2005). Ello se vería reflejado en la construcción de conocimiento de forma permanente por la comunidad educativa, respecto del saber (Abell, 2008). Hasta aquí se considera la construcción epistemológica de la pedagogía desde el saber; no obstante, otra definición en la que profundizaremos construye un concepto pedagógico desde el discurso.

\section{Pedagogía concebida desde el discurso}

Establecer una episteme de la pedagogía desde el discurso, sería situarla desde un discurso conceptual, desde una consecuencia respecto de la reflexión sobre la formación, en relación con una intencionalidad que estará dada en un escenario formal y no formal con cada uno de los agentes educativos, es decir, docentes y estudiantes (Zapata, 2006).

En la concepción pedagógica de Comenio se evidencia de forma explícita una pedagogía discursiva que logra generar conceptualizaciones que tienen un método y una didáctica que funda la pedagogía de la modernidad que se manifiesta a través de una visión cientificista de la educación que, como bien señala Herbart, tendría su base en la instrucción, la cual es observable en otras posiciones que la instituyen como la única vía que tiene el hombre para ser hombre y poder sobresalir ante los demás (Comenio, 1982).

Situarse en una educación con base en la comprobación, implica advertir una pedagogía confinada a un carácter científico. Debido a ello, será Herbart uno de los primeros intelectuales que instala la pedagogía de las ciencias, concibiendo al ser humano como un objeto perfectible a través de la instrucción, ubicando al estudiante en el centro de todo proceso de educativo. En este sentido, lo importante no sería el discurso pedagógico, sino más bien el saber pedagógico a través de un método. Desde este enfoque, comienza un cambio en la teoría del conocimiento en relación con el campo educativo (Sanhueza, 2012). Ello, implica concebir al ser humano como un objeto, es decir, la cosificación ya no sólo del conocimiento, sino también de la persona.

Entender una pedagogía de la ciencia remite, necesariamente, a una tradición que se basa en la instrucción y en el convencimiento de que es necesario encapsular los conocimientos para aportar a una sociedad industrializada. Lo anterior, en relación a los intereses del Estado (Herbart, 1935). Esta visión sobre la pedagogía comprende a la persona que aprende, como un sujeto que estará en una relación de tipo asimétrica, en la cual todo tipo de poder se establecerá en base a un sujeto que posee conocimientos y otro que no (Herbart, 1945).

La pedagogía de la ciencia sitúa el proceso de enseñanza-aprendizaje desde el foco del objeto y su ocupación como el fundamento de los intereses múltiples, que decantarán en el objetivo de esta pedagogía, donde el proceso de aprender se valida a través de la instrucción (Herbart, 1935). 
Esta visión de educación lo que evidencia es la cosificación de la persona y en sí mismo es un fin para alcanzar los objetivos últimos de una sociedad moderna e industrializada. La educación al servicio de los intereses económicos del hombre para el grupo de dominación, que no puede instituir otro dominio más que el del hombre por el hombre.

La pretensión de este tipo de modelos educativos busca la cosificación de los sujetos como entidades de intercambio comercial en la que se puede crear mano de obra calificada, que servirá a los obreros calificados en una sociedad dominada por una educación de mercado (Lane y Kinser, 2012).

Este tipo de visiones en relación a lo pedagógico como discurso, de alguna u otra forma, estarían cruzadas con el saber pedagógico que se ve implicado en la formación docente que le atribuye sentido al discurso disciplinario (Philipsen, 2007).

Saber y discurso pedagógico se encuentren imbricados y se funden en la práctica que proporciona evidencia de que las relaciones socioculturales serían la forma en que se expresan estos discursos pedagógicos. Lo anterior se vuelca en la práctica, aunque sean muy distintas, una tributa en la otra, pues práctica es un discurso y viceversa (Zapata, 2006), aunque ocurra resistencia del estudiante entorno al discurso pedagógico (Singh, 2001). Sin embargo, otras definiciones dejan de lado el saber y el discurso para situarse en una concepción que aborda la pedagogía desde una disciplina.

\section{Pedagogía desde el campo disciplinar}

La pedagogía considerada como disciplina define, aplica y experimenta los diversos conocimientos y saberes acerca de ellos, específicamente, en una determinada cultura (Zuluaga, 1987), por ende se enfoca en la acción pedagógica a través de los saberes inherentes en una cultura.

Esta conceptualización de la pedagogía como disciplina reconstructiva se plantea como una instancia, en la cual se considera el saber intrínseco cultural como punto de partida y complemento en relación a los saberes que podría acompañar en su desarrollo social y científico (Mockus, 1995). Este tipo de pedagogía hace explícita la necesidad de un educador en relación con su formación ética y moral, que asuma su práctica con sentido en la liberación del conocimiento, haciendo un uso formal bajo un marco de actuación dogmático de la acción educativa (Herrera, 2008). En este sentido, la axiología de la educación se acerca bastante a este proyecto, pues significa poner en el centro una educación en valores, los cuales serán el sostén de toda práctica; no obstante, los juicios emitidos estén solapados de forma permanente. La interculturalidad es un hecho, que implica la existencia y convivencia de valores y de modos de vida distintos; pero además es un ejercicio de voluntades y un compromiso moral, porque los conocimientos culturales de por sí, no mejoran necesariamente la comprensión del otro, ni la relación (Touriñán, 2005).

Finalmente entenderemos la pedagogía como una disciplina reconstructiva, contextualizada como fenómeno cultural, encargada de explicitar lo implícito para luego transformarlo. Es decir, el foco estaría en relevar la buena práctica de la enseñanza y el discurso fundamentado acerca de cómo enseñar de forma adecuada. Lo anterior nos remite a una disciplina que se identifica y se concibe desde la misma práctica educativa. Ello permitiría atender cada una de las dimensiones pedagógicas involucradas en el proceso de enseñanza -aprendizaje (Chiappe Laverde, 2009).

Situarse en una educación que tenga relación con los valores de una persona significa tomar conciencia que estamos ante una sociedad que debe pensarse en relación a una 
moral y un sentido de moralidad y ética que la concierne en aspectos que sean relativos a un individuo en relación a una formación que aliente a una mejor sociedad (Olivé, 1993).

Esta visión acerca de la pedagogía la constituye en una epistemología que estaría explicada en su saber y método de cómo abordar el proceso de enseñanza, de igual forma no ligitima el discurso pedagógico como una construcción epistemológica de la pedagogía, pues lo enmarca dentro de las herramientas que tiene el docente para enfrentar la acción pedagógica en la sala de clases.

El saber pedagógico que está en relación con la concepción de la pedagogía como disciplina reconstructiva estaría en relación con un saber que se reconstruye de forma continua, demandando procesos de investigación en el aula, sólo desde allí se podrá establecer un razonamiento y una comprensión de la praxis pedagógica (Rojas, 2005).

\section{Pedagogía como conjunto de concepciones implícitas}

La pedagogía puede definirse como un sistema de mensajes implícitos, que tendrán el propósito de regular las relaciones entre quienes participan en esas prácticas (Bernstein, 1958; Mockus, 1995).

Bien es sabido que en el sistema educativo se emplean de forma constante distintas metodologías que, de alguna u otra forma, aseguran ciertas técnicas y procedimientos que proporcionan el sostén de un proceso educativo, pero la elección de una u otra didáctica está supeditada a funciones y finalidades que, justamente, se encuentran de forma implícita en el acto pedagógico, que en muchos casos hasta se ve explicitado en las concepciones que tienen los propios estudiantes en el proceso de aprendizaje (De la Cruz y Pozo, 2001).

Lo anterior nos remite, necesariamente, a evidenciar esos criterios que pudiesen estar en un nivel implícito, explícito o relacional que hacen que la pedagogía se conciba y se construya, epistemológicamente, de una u otra forma. Al respecto, Pozo y Scheuer (1999), han estudiado, ampliamente, las concepciones de los profesores sobre la pedagogía. Estas concepciones las han entendido desde el marco de las teorías implícitas (Pozo et al., 2006).

Es preciso distinguir tres niveles representacionales en las concepciones, los que para efectos de este ensayo se considerarán. En el primer nivel estarían las respuestas de los sujetos ante una situación, las cuales son explícitas, respecto de sus creencias, juicios e interpretaciones (Pozo y Scheuer, 1999).

Un segundo nivel estará dado por aquellas representaciones que se encuentran anquilosadas y con una escasa posibilidad de poder acceder a ellas de manera consciente. En este sentido, se hallarían las teorías de dominio o los mensajes implícitos. Este tipo de teorías sólo pueden ser inferidas y atañerían a las regularidades conceptuales que están subsumidas en las creencias de las representaciones explícitas. Al respecto, las teorías de dominio estarían en directa relación con los rasgos estáticos de los modelos mentales elaborados en diferentes contextos para un mismo ámbito de conocimiento (Op cit.).

Para efectos de este ensayo se considerarán las concepciones implícitas, las cuales se estructurarían a partir de una serie de supuestos implícitos que constituirían una teoría implícita. Estas teorías estarían compuestas a partir de una serie de restricciones en el procesamiento de la información que restringirían no sólo la información procesada, sino 
también las relaciones establecidas entre los elementos de esa información (Pozo y Scheuer, 1999).

Las concepciones entendidas como un proceso mental que se integran al conocimiento en la medida en que el sujeto las va estructurando (Giordan y De Vecchi, 1995) estarían directamente relacionadas con el proceso de intercambio y adquisición de conocimiento en el proceso formativo y de la acción del pedagogo. En este caso se está ante el tercer tipo de concepción, la teoría relacional. En tanto, estas concepciones tienen sus orígenes en procesos socioculturales, que se encuentran en la base de los intercambios psicosociales producidos en el campo de acción de un pedagogo (Rodrigo, Rodríguez y Marrero, 1993).

En este sentido, el profesor se va estructurando en base a estas múltiples relaciones socioculturales que, asientan sus visiones y creencias del mundo en relación a su campo de desarrollo profesional. En este proceso, el pedagogo estructura su cosmovisión personal y profesional sobre las distintas dimensiones que recoge su accionar en una institución educativa. A este respecto, Pozo y Scheuer (1999) señala que "(...) todos tenemos creencias o teorías profundamente asumidas y tal vez nunca discutidas, sobre lo que es aprender y enseñar" (p. 34). Estas creencias rigen nuestras acciones al punto de constituir un verdadero currículo oculto que guía nuestras prácticas educativas. Justamente, este tipo de creencias son las que podrían ser un facilitador o un obstaculizador en el momento de alcanzar el logro de los aprendizajes y llevar un proceso de enseñanza, los cuales son eje fundamental para construir una epistemología de la pedagogía.

Las concepciones de los pedagogos han sido un tema tratado que, actualmente, tiene su foco en dos perspectivas. La primera, cognitiva, trata acerca de las operaciones mentales de los pedagogos en los diferentes momentos de su quehacer pedagógico. La segunda, alternativa, señala que la enseñanza no podría estar alejada de la intención y la cultura que antecede al profesor, constituyéndose en el currículum oculto de este (Perafán y Adúriz-Bravo, 2002).

Los mensajes implícitos que tienen los pedagogos en muchas ocasiones se han mantenido anquilosados y son muy difíciles de movilizar, debido a los procesos socioculturales vividos desde lo personal y lo escolar, puesto que son marcadoras en las prácticas que desarrollen los docentes.

Los mensajes implícitos que tienen los docentes vienen de una formación que se origina en una serie de teorías implícitas, en las cuales el pedagogo en formación se muestra muy confiado sobre los aspectos afectivos y no presta mucha atención a los cognitivos en el proceso de enseñanza- aprendizaje, de igual forma, se siente tentado a explicar las acciones pedagógicas en la experiencia cotidiana y de la externalización de las dificultades y que no pueden cambiarlas (Woolfolk, Davis \& Pape, 2006). Lo anterior es totalmente opuesto a una visión constructivista del proceso de enseñanza- aprendizaje (Brooks \& Brooks, 1993; Brown, 1994).

\section{Conclusiones}

En el contexto educativo actual, el profesor tiene un rol de mediador entre aquello que se aprende y se enseña. En este proceso, el profesor considera algunas metodologías para desarrollar su trabajo y alcanzar aquellos objetivos propuestos por la institución o el Estado, primando una educación empírica. Sin embargo, no es que esta categorización de su acción pedagógica quede clara donde el profesor tenga una teoría y una construcción del saber pedagógico que defina y maneje. Esta situación descrita 
es la que genera confusiones de aplicación de didácticas y formas de evaluación que, en muchos de los casos están bajo la intuición o en su defecto bajo una tradición que se incrusta en la cultura escolar.

La escolarización del sistema educativo ha permitido el encapsulamiento de los saberes, los cuales se traducen en procesos de transmisión de información validada desde arribaEstado- y no desde abajo- comunidad educativa.

Cada uno de los procesos pedagógicos instalados en la sala de clase no están pensados desde una pedagogía que cuestiona los procesos, instando a cada uno de los agentes educativos a repensar y reflexionar sobre su quehacer pedagógico, por lo tanto, la pregunta que cabe hacerse es ¿hay espacio para pensar en pedagogía en la comunidad educativa? Ante esta pregunta una de las respuestas que emerge es que cada uno de los agentes educativos está preparado y sus expectativas del proceso de enseñanza -aprendizaje están hechos para la instrumentalización.

En este sentido, toda institución formadora de profesores debiese tener como objetivo esencial el saber pedagógico (Bedoya, 2003). Lo anterior pudiese proveer a toda institución de educación superior a una identidad propia de estas comunidades universitarias centradas en el saber, no por el conocimiento, sino por un entendimiento cabal sobre los procesos formativos en los que se instala un futuro profesor. La realidad universitaria evidencia el propósito de producir conocimiento, sin generar ningún tipo de cuestionamiento respecto de las condiciones en las que se desarrolla o se elabora ese conocimiento. Lo anterior deja de manifiesto la poca discusión pedagógica en las instituciones educativas, pues no hay tal tipo de reflexión (Ahumada, 2001).

Una propuesta en relación con la situación que vive la pedagogía debiese estar alineada en relación con un estatus teórico, investigativo y práctico-metodológico, las que al instalarse en las facultades de pedagogías de una institución universitaria le podrían asignar su carácter o sello, según se sientan identificadas con ellas. Esta propuesta no se agota solo en un ámbito praxiológica de la pedagogía. En este sentido, esta propuesta debiera estar vinculada a un estudio crítico epistemológico que tenga como finalidad evidenciar de qué forma lo pedagógico ha estado presente en el sistema educativo a partir de la instalación en la modernidad.

El discurso pedagógico tiene por objeto la relación pedagógica, la cual se manifiesta como aquellas actuaciones que se establecen en las relaciones sociales, las cuales están implicadas en el proceso histórico social instaladas en la práctica pedagógica.

El estudio de las relaciones pedagógicas y su actuación histórica y dialectal serán de vital importancia para comprender el proceso educativo en un contexto de relaciones de dominación. La comprensión de este escenario educativo histórico- político proporcionará las claves para evidenciar toda ideología pedagógica que subyace en cada uno de los procesos educativos o aquellas que representan a un grupo o clase social en el poder.

En el ámbito educativo muchas de las relaciones que se establecen están imbricadas entre la transmisión y adquisición de saberes teóricos y prácticos, esta situación según Ahumada (2001) no dejaría espacio a la reflexión pedagógica y a la pregunta desde dónde surgen dichos saberes, cuestión vital para el proceso de formación pedagógica y al responderse podría incluso generar una implicación en la apropiación de sus propias prácticas y la propia transformación de estas (Lunenberg, Korthagen \& Swennen, 2007).

\section{Referencias}


Abell, S. K. (2008). Twenty years later: Does pedagogical content knowledge remain a useful idea? International Journal of Science Education, 30(10), 1405-1416.

Ahumada, P. (2001). La evaluación en una concepción de aprendizaje significativo. Valparaíso: Ediciones Universitarias Universidad Católica de Valparaíso.

Arboleda, L.C. y Castrillón, G. (2003). Educación Matemática, Pedagogía y Didáctica. En Educación y formación del pensamiento científico, Bogotá: ICFES-Universidad del Valle.

Bedoya, J. I. (2003). Epistemología y pedagogía. Ensayo histórico crítico sobre el objeto y método pedagógicos. Bogotá: Ecoe Ediciones.

Beillerot, J. (1998), Saber y relación con el saber, Buenos Aires, Paidós.

Bernstein, B. (1958). Some sociological determinants of perception: an enquiry into sub-cultural differences [Algunos determinantes sociológicos de la percepción: estudio de las diferencias subculturales]. British journal of sociology (Londres), vol. $9, \mathrm{n}^{\circ} 1$, págs. 159-174.

Brooks, J. G., y Brooks, M. G. (1993). The case of constructivist classrooms. ASCD: Alexandria, VI.

Brown A. L. (1994) The advancement of learning. Educational Researcher, n. 23, 4-12.

Comenio, Juan Amós (1982), Didáctica magna, México, Porrúa ("Sepan cuantos...", 167)

Chiappe Laverde, A. (2009). Acerca de lo pedagógico en los objetos de aprendizaje-reflexiones conceptuales hacia la construcción de su estructura teórica. Estudios pedagógicos (Valdivia), 35(1), 261272

De la cruz, M., J. I. Pozo. (2001). "Concepciones de enseñanza de profesores universitarios". Actas Pedagógicas, Año 2, 1: 5-15.

Foucault, M. (1982). La arqueología del saber. México: Siglo XXI.

Foucault, M. (1997). Las palabras y las cosas. Madrid: Siglo XXI.

Giordan, A. y De Vecchi,G. (1995). Los orígenes del saber. Sevilla: Diada.

Herbart, J. (1935) Pedagogía General. Derivada del fin de la educación. Madrid, Espasa-Calpe.

Herbart, J. (1945) Bosquejo para un Curso de Pedagogía. Madrid, Espasa-Calpe.

Hermoza, L. P. (2011). Universidad peruana: tendencias, tensiones y resultados. Innovación Educativa, 11(57), 99-111.

Herrera, M. A. P. (2008). Evolución de la práctica pedagógica como dispositivo escolar y discursivo en la educación artística-musical. Universidad de Caldas.

Lane, J., \& Kinser, K. (2012, septiembre 29). MOOC's and the McDonaldization of global higher education. world.edu. Recuperado a partir de http://world.edu/moocs-and-themcdonaldization-ofglobal-higher-education/
Latorre, M. (2002). Saber pedagógico en uso-, caracterización del saber actuante en las prácticas pedagógicas. Tesis Doctoral. Pontificia Universidad Católica de Chile

Latorre, M. (2004). Aportes para el análisis de las racionalidades presentes en las prácticas pedagógicas. Estudios Pedagógicos (30), 75-91.

Loayza, Norman (2009) "Globalización y informalidad: dos retos para el desarrollo y la integración" in BBVA Las Multiples Caras de la globalización. s/l: BBVA, 116-125

Lunenberg, M. Korthagen, F. \& Swennen, A. (2007). The teacher educator as a role model. Teaching and Teacher Education, n. 23, 586-601

Maturrano, E. F. L. (2009). La naturaleza epistemológica de la Pedagogía: una resignificación cuantitativacualitativa. Investigación Educativa, 13(24), 169-177.

Mena, M. I. y Romagnoli C. (1993). Convivencia social en la Enseñanza Media. Santiago: CPU.

Mockus, A., Hernández, C.A., Granés, J.,Charum, J. \& Castro M.C. (1995): Las fronteras de la Escuela. Articulaciones entre conocimiento escolar y conocimiento extraescolar. Bogotá: Cooperativa Editorial Magisterio.

Mockus, Antanas. (1995). Lugar de la pedagogía en las universidades. Documentos para la Reforma Académica. Universidad Nacional de Colombia, Bogotá.

Muñoz, J, Quintero, J. y Munévar, R. (2002). Experiencias en investigación-acción-reflexión con educadores en proceso de formación en Colombia. Revista Electrónica de Investigación Educativa, 4, 15-21

Noguera, C. (2005). La pedagogía como "saber sometido": un análisis del trabajo arqueológico y genealógico sobre el saber pedagógico en Colombia. En O. Zuluaga y C. Noguera, Foucault. La educación y la pedagogía (pp. 39-69). Bogotá: Magisterio.

Olivé, L. (Comp.) (1993) Ética y diversidad cultural. México, Fondo de Cultura Económica.

Patiño-Garzón, L., \& Rojas-Betancur, M. (2009). Subjetividad y subjetivación de las prácticas pedagógicas en la universidad. Educación y Educadores, 12(1), 93-105.

Perafán, G. y Adúriz-Bravo, A. (2002). Pensamiento y conocimiento de los profesores. Bogotá: Universidad Pedagógica NacionalColciencias.

Philipsen, G. (2007). The early career rise of "speech" in some disciplinary discourse, 1914-1946. Quarterly Journal of Speech, 93(3), 352-354

Pozo, J, I., Scheuer, N., Mateos, M. \& Pérez Echeverría, M. (2006). Las concepciones de los profesores de educación primaria sobre la enseñanza y el aprendizaje. En Pozo, J., et al., (2006). Nuevas formas de pensar la enseñanza y el aprendizaje. Barcelona: Grao.

Pozo, J., Scheuer, N., Pérez Echeverría, M., Mateos, M, Martín, E \& de la Cruz, E., (2001). Nuevas formas de pensar la enseñanza y el aprendizaje (pp. 171-188). Graó: Barcelona. 
Pozo, J.I. y Scheuer, N. (1999). "Las concepciones sobre el aprendizaje como teorías implícitas" (pp. ). En j.i. pozo y c. monereo (eds.). El aprendizaje estratégico. Madrid: Santillana/aula xxi.

Rodrigo, M. J., Rodríguez, A. y Marrero, J. (1993). Las teorías implícitas: una aproximación al conocimiento cotidiano. Madrid. Aprendizaje visor.

Rojas, M. (2005) Investigar la investigación. La práctica docente en la enseñanza de la metodología de la investigación en la universidad. Universidad de Ibagué.

Sanhueza, C. (2012). El objetivo del instituto pedagógico no es el de formar geógrafos. hans steffen y la transferencia del saber geográfico alemán a chile. 1893-1907. HISTORIA No 45, vol. I, enero-junio 2012: 171-197.

Singh, Parlo (2001) Pedagogic Discourses and Student Resistance in Australian Secondary Schools. In et al, Morais (Ed.) Towards A Sociology Of Pedagogy: The Contribution Of Basil Bernstein To Research. Peter Lang Publishing, Inc., New York, USA, pp. 251276.

Tamayo Valencia, Luis Alfonso. (2007). Tendencias De La Pedagogía En Colombia. En: latinoam.estud.educ. Manizales (Colombia), 3 (1): $65-76$.
Tardif, M. (2004). Los saberes del docente. Madrid: Narcea

Touriñán, J. M. (2005) "Posibilidad y necesidad de la educación en valores". Proyecto Educación en Valores. Atei. (www.ateiamerica.com)

Woolfolk Hoy, A., Davis, H., \& Pape, S. (2006). Teachers' knowledge, beliefs, and thinking. En P. A. Alexander \& P. H, Winne (Eds.), Handbook of Educational Psychology (pp. 715737). Mahwah, NJ: Lawrence Erlbaum.

Zapata, V. (2006) La memoria activa del saber pedagógico en la contemporaneidad. Historia de la Educación, Salamanca, 25, 533552.

Zuluaga, O. (1999) Pedagogía e Historia: la historicidad de la pedagogía, la enseñanza, un objeto de saber. Recuperado de https://www.udesa.edu.ar/files/ESCEDU/DT/DT10-GAINZA.PDF • Gainza, V., \& Hemsy, V. (2003). La educación musical entre dos siglos.

Zuluaga, O. (1999): «El florecimiento de las investigaciones pedagógicas», en Pedagogía, discurso y poder, Bogotá, Corprodic.

Zuluaga, O. L. (1987) Pedagogía e historia. Editorial Foro. Bogotá. 